DOI: 10.1515/awutp -2015-0209

\title{
RADIOFREQUENCY ELECTROMAGNETIC FIELD MAP OF TIMISOARA
}

\author{
N. Stefu*, I. Solyom, A. Arama \\ Faculty of Physics, West University of Timisoara,Bd. V. Parvan nr. 4, 300223, Timisoara, ROMANIA
}

\begin{abstract}
Article Info
Received: 19.12 .2014

Accepted: 11.02.2015

Keywords:

Electromagnetic field map, electromagnetic power density measurements.
\end{abstract}

\begin{abstract}
There are many electromagnetic field (EMF) sources nowadays acting simultaneously, especially in urban areas, making the theoretical estimation of electromagnetic power at ground level very difficult. This paper reports on EMF maps built with measurements collected in Timisoara, at various radiofrequencies. A grid of $15 \times 15$ squares was built (approximate resolution $400 \mathrm{~m} \times 400 \mathrm{~m}$ ) and measurements of the average and maximum values of the electric field $\mathrm{E}$, magnetic field $\mathrm{H}$ and total power density $\mathrm{S}$ at $0.9,1.8$ and $2.4 \mathrm{GHz}$ were collected in every node of the grid. Positions of the nodes in terms of latitude and longitude were also collected. Maps were built presenting the spatial distribution of the measured quantities over Timisoara. Potential influences of EMF on public health are discussed.
\end{abstract}

\section{Introduction}

Radiofrequency electromagnetic field (EMF) exposure of population has increased in last decades due to rapid development of new wireless and communication technologies [13]. In urban areas, mainly, there are several EMF sources acting simultaneously, hence a theoretical estimation of electromagnetic power at ground level can be very difficult. Nevertheless, the population should be aware of the EMF it is surrounded by, and of the health hazard it poses.

The range of devices working with EMF in radio and microwave frequencies is very wide nowadays:

-WLAN: $2.4 \mathrm{GHz}, 3.6 \mathrm{GHz}, 5 \mathrm{GHz}$ si $60 \mathrm{GHz}$;

- Microwave ovens - $2.45 \mathrm{GHz}$;

- wireless sensors- $915 \mathrm{MHz}$ and $2.450 \mathrm{GHz}$;

• cordless phones - $915 \mathrm{MHz}, 2.450 \mathrm{GHz}$ and $5.800 \mathrm{GHz}$;

- Bluetooth devices $2.4-2.4835 \mathrm{GHz}$; 
- car alarm (internal movement sensors) - $2.45 \mathrm{GHz}$, with a power of $500 \mathrm{~mW}$;

- Mobile phone operators (in Romania): Telekom Romania-GSM-900 MHz, GSM/LTE-1800 GHz, 3G (UMTS)-2100 MHz; Orange and Vodafone - GSM 900 MHz, DCS-1800 MHz, 3G (UMTS)-2100 MHz; Digi (RDS/RCS) - 3G (UMTS)-2100 $\mathrm{MHz}$.

This paper aims to offer some information on this issue, by presenting some electromagnetic field maps built with measurements collected in Timisoara, at various radiofrequencies, and discussing potential influences of EMF on public health.

\section{Data}

According to ICNIRP regulations [4], for the far field region, where the electromagnetic waves can be considered as plane waves, the power density $S\left[\mathrm{~W} / \mathrm{m}^{2}\right]$ representing the electromagnetic power per unit area normal to the direction of propagation of electromagnetic waves is recommended to be measured. From the power density, the electric $(E)$ and magnetic $(H)$ field intensities can be determined.

In order to collect the electromagnetic field data, a grid of 225 squares (15x15) was superimposed over the city map, and measurements were performed in each node, within city limits (Fig. 1). An approximate resolution of 400m x400m was achieved, and 210 sets of data were collected, for each of the three frequencies investigated: GSM-900 MHz, GSM-1800 MHz and $2100-2450 \mathrm{MHz}$ (UMTS-ISM). Each set of data consists of the GPS coordinates of the point, the average and maximum values of the electric field $E$, magnetic field $H$ and total power density $S$.

Electromagnetic field measurements were performed with a Radio Frequency EMF Strength Meter, properly calibrated for each frequency (Fig. 2). The calibration factors for $2100 \mathrm{MHz}$ and $2450 \mathrm{MHz}$ are very close, so the measured values were assigned to the 21002450 band. Maps were built using Surfer11 Software and results are presented in Figs. 3-5. A digital map of Timisoara was used as a base layer, over which a contour map obtained by interpolation of the measured data was superimposed for each set of data. The Kriging gridding method [5] was used for interpolation. 


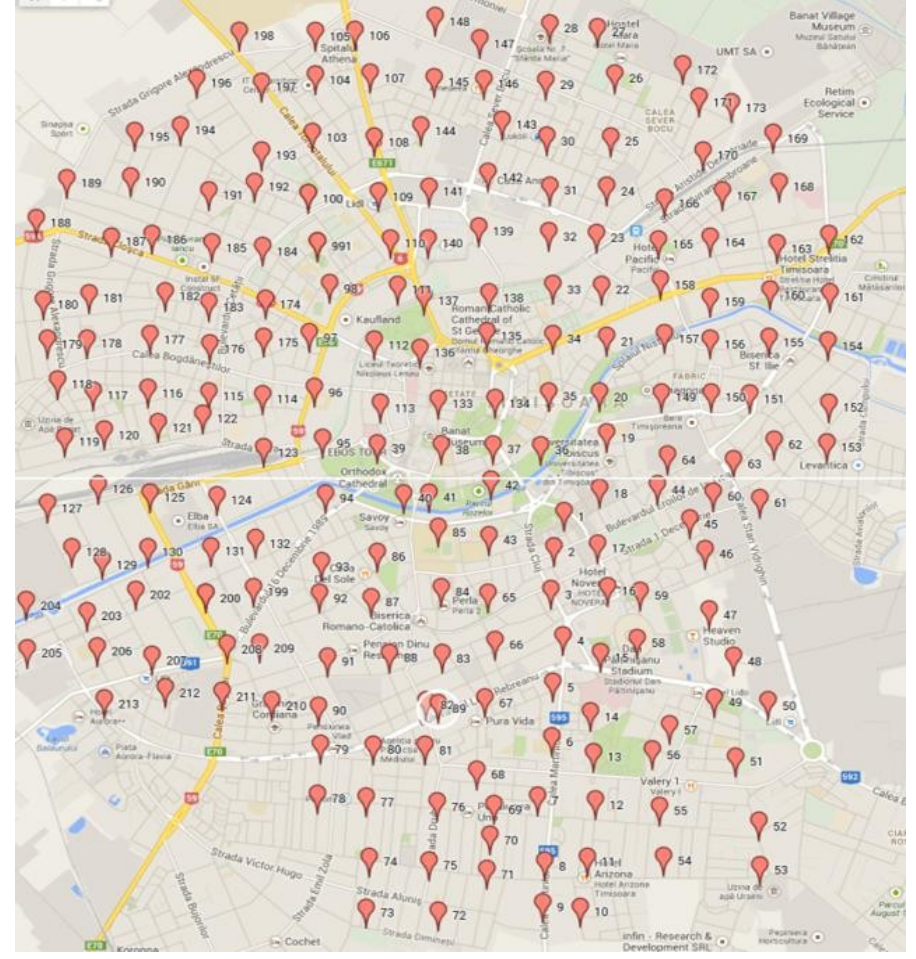

Fig 1. Map of the city of Timisoara with the points of measurement

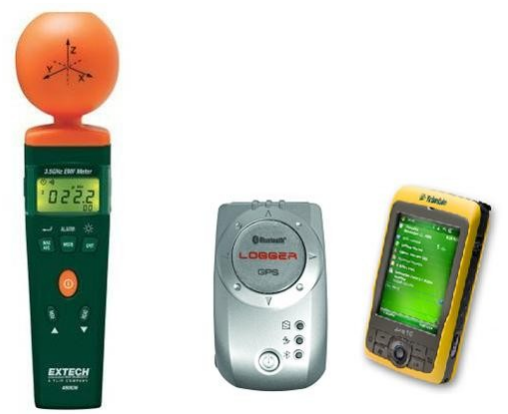

Fig 2. EXTECH Instruments RF EMF Strength Meter and GPS with data logger used in measurements

\section{Results}

The resulting EMF maps are presented in figures 3-5, for the measured mean values of electric field intensity $E_{\text {mean }}$, the magnetic field intensity $H_{\text {mean }}$, and the mean and maximum total electromagnetic power density $S$, for each of the three frequencies chosen: GSM-900 MHz, GSM-1800 MHz and $2100-2450 \mathrm{MHz}$ (UMTS-ISM).

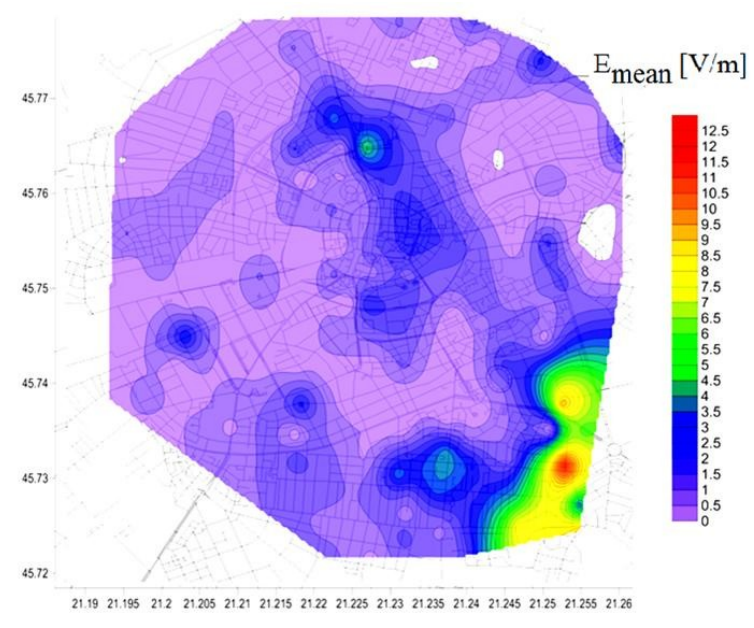

a)

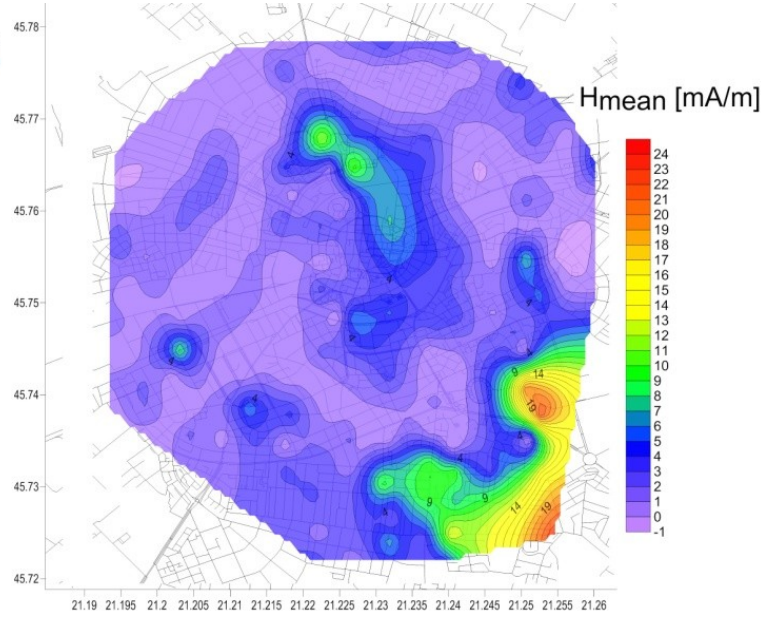

b) 


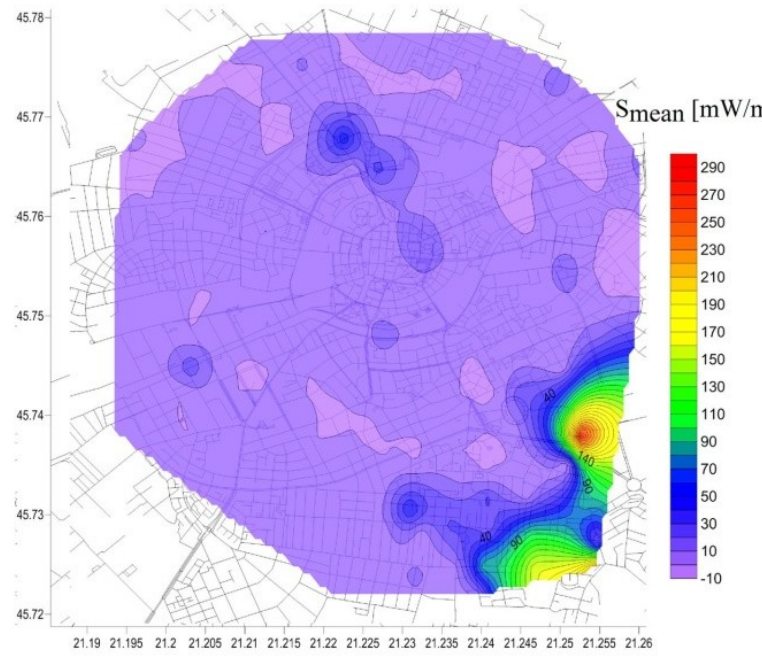

c)

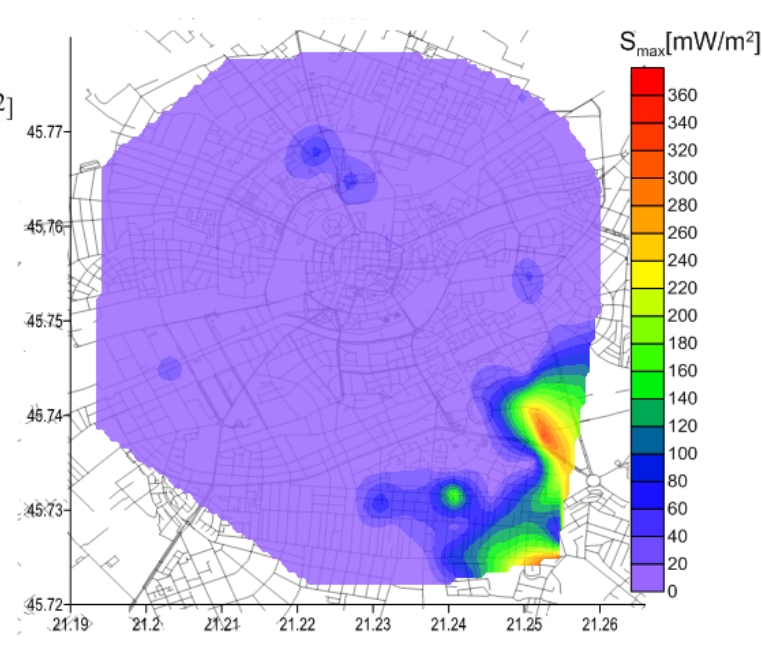

d)

Fig. 3. Results for frequency GSM-900 MHz (reference levels [4]: $E<41 \mathrm{~V} / \mathrm{m} ; H<111 \mathrm{~mA} / \mathrm{m} ; S<4500$ $\mathrm{mW} / \mathrm{m}^{2}$ )

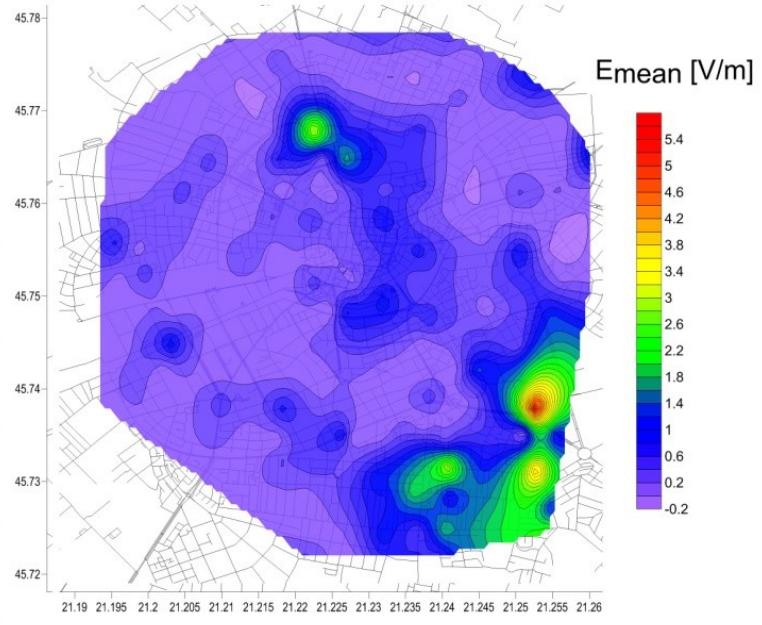

a)

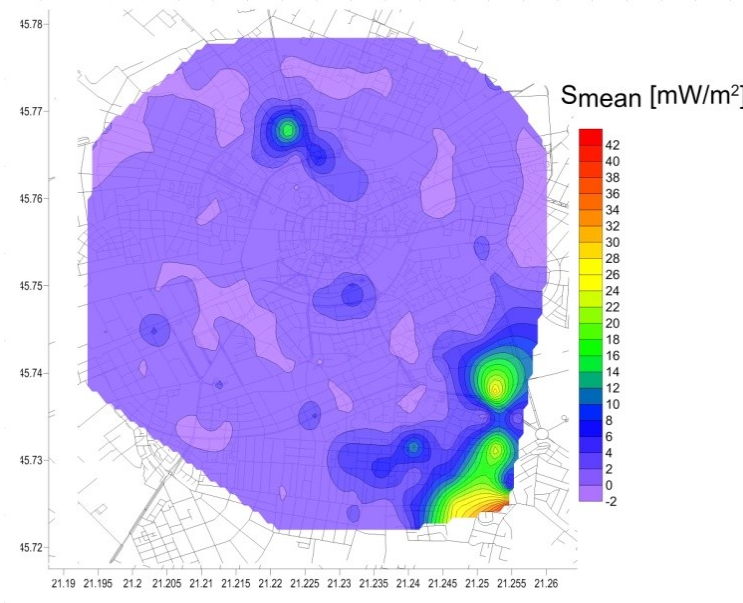

c)

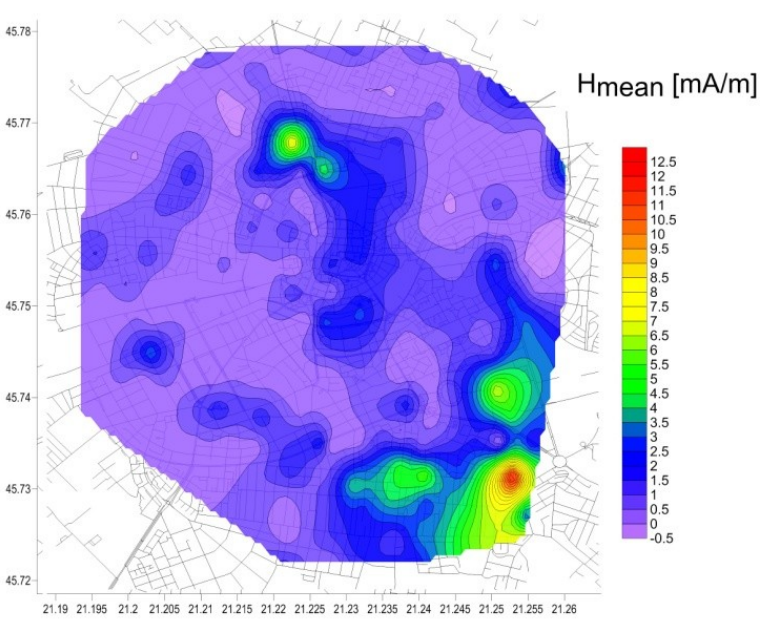

b)

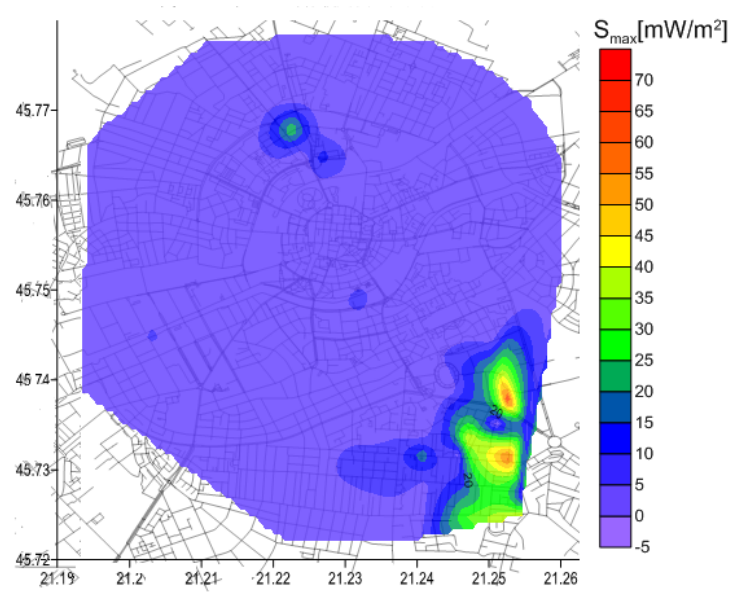

d)

Fig. 4. Results for frequency GSM-1800 MHz (reference levels [4]: $E<58 \mathrm{~V} / \mathrm{m} ; H<157 \mathrm{~mA} / \mathrm{m} ; S<9000$ $\left.\mathrm{mW} / \mathrm{m}^{2}\right)$ 


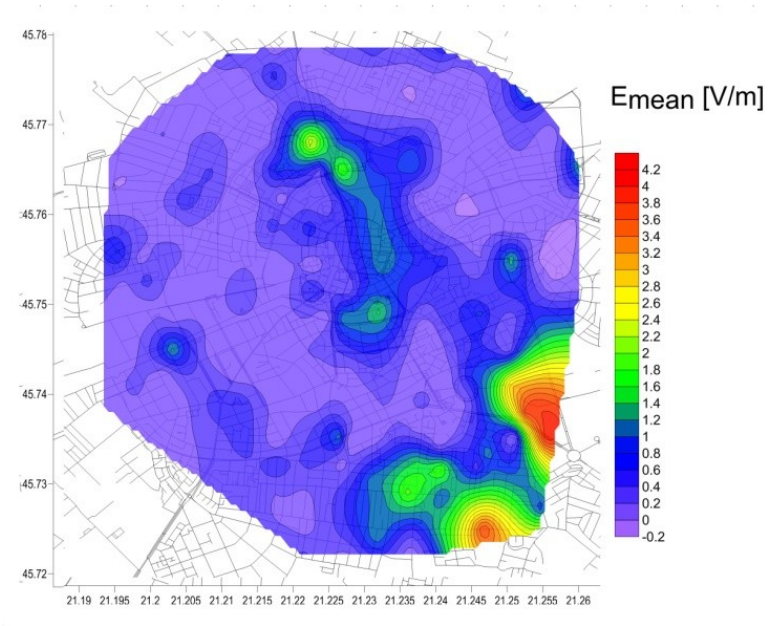

a)

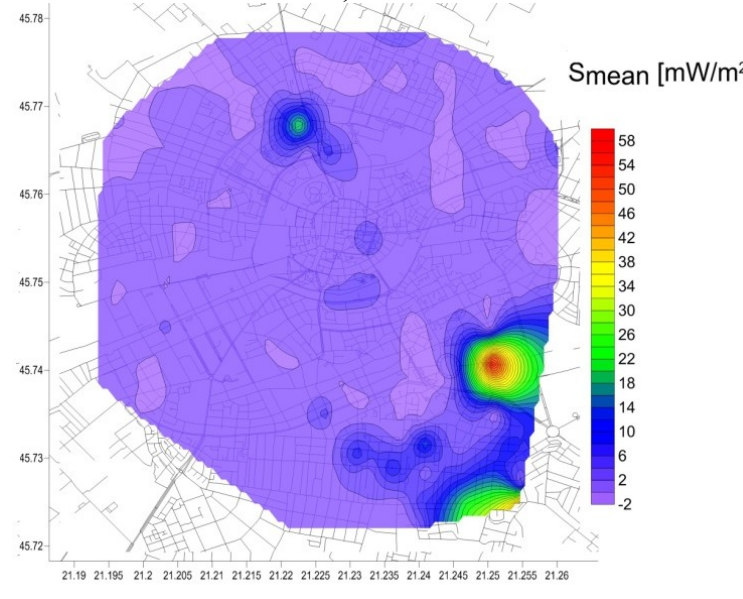

c)

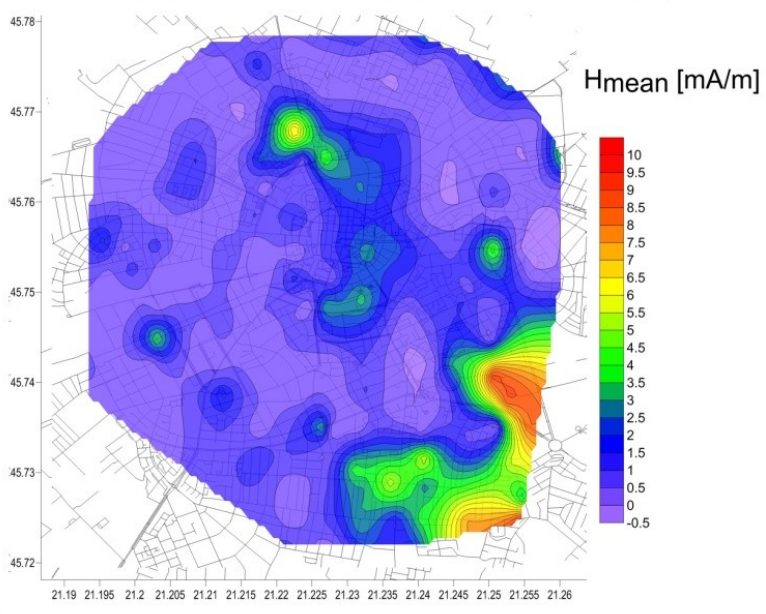

b)

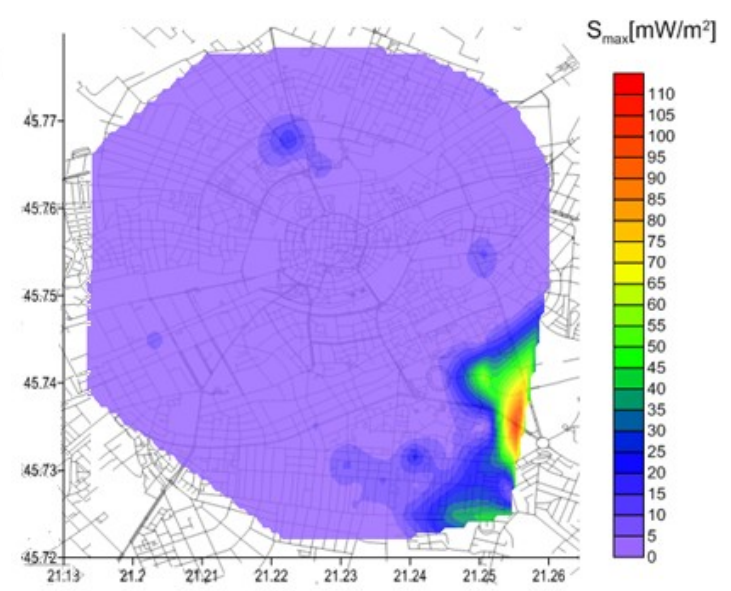

d)

Fig. 5. Results for frequencies $2100-2450 \mathrm{MHz}$ (UMTS-ISM) (reference levels [4]: $E<61 \mathrm{~V} / \mathrm{m} ; H<160$ $\left.m A / m ; S<10000 \mathrm{~mW} / \mathrm{m}^{2}\right)$

\section{Discussions}

The reference levels for electric, magnetic and electromagnetic fields for frequencies between 0-300 GHz, according to "ICNIRP guidelines for limiting exposure to time-varying electric, magnetic and electromagnetic fields (up to $300 \mathrm{GHz}$ )" [4], calculated for the three frequencies analyzed here, are written in each figure caption. The maximum permissible exposure in terms of power density given in [6] is $2 \mathrm{~W} / \mathrm{m}^{2}$ between 30 and $400 \mathrm{MHz}$, and 10 $\mathrm{W} / \mathrm{m}^{2}$ between 400 and $2000 \mathrm{MHz}$. For frequencies greater than $2000 \mathrm{MHz}$, the maximum permissible exposure is also $10 \mathrm{~W} / \mathrm{m}^{2}$.

Taking these values into account, one can notice in figures 3-5 that there are no electric field intensity, magnetic field intensity and power density mean values exceeding these reference levels allover Timisoara, not even in the South-Eastern part where a powerful RF antenna is located (Urseni). More precisely, for GSM-900 MHz, the electric and magnetic field intensities are at least 4 times lower than the reference level, and the electromagnetic 
power density $S$ is at least 15 times lower. For the higher frequencies, the ratio gets even higher, to over 10 for $E$ and $H$ and over 150 for $S$. As for the maximum values of $S$, it can also be seen that they are over 10 times smaller than the reference value for GSM-900 $\mathrm{MHz}$ and over 100 times smaller for the higher frequencies.

Some typical values reported in literature are: the peak value of a WLAN terminal is $200 \mathrm{~mW}$, but close to a WLAN system exposure is typically below $500 \mathrm{~mW} / \mathrm{m}^{2}$; the range of exposure of the general population due to GSM signals is typically between some hundred $\mathrm{nW} / \mathrm{m}^{2}$ and some tens of $\mathrm{mW} / \mathrm{m}^{2}[7]$; under an antenna, the values $\mathrm{S}_{\text {mean }}=0.310 \mathrm{~W} / \mathrm{m}^{2}$ and $\mathrm{S}_{\max }=$ $0,335 \mathrm{~W} / \mathrm{m}^{2}$ were reported in [8].

For UMTS (2100 MHz), the available measurements are limited, and so far the traffic is rather low compared to GSM. Values slightly over $1 \mathrm{~mW} / \mathrm{m}^{2}$ have been measured in a few cases, while minimum levels are a few hundred $\mathrm{nW} / \mathrm{m}^{2}$ [7].

Other important RF sources are radio broadcasting systems (AM and FM). The maximum values measured in areas accessible for the public are typically below $10 \mathrm{~mW} / \mathrm{m}^{2}$. Close to the fences of very powerful transmitters, exposure of about $300 \mathrm{~mW} / \mathrm{m}^{2}$ can be expected in some cases [7]. This is in accordance with the highest values measured by us in the area surrounding the antenna, at GSM-900 MHz.

It was estimated in [7] that the maximum power density of a beam reaching ground level $50 \mathrm{~m}$ from an antenna mounted at a height of $10 \mathrm{~m}$ would be around $100 \mathrm{~mW} / \mathrm{m}^{2}$. By comparing our measured values to these reported ones, one can see that the highest value of $S_{\text {mean }}$ at GSM-1800 MHz and the one in UMTS range, measured near the antenna located in the $\mathrm{SE}$, is about half the value reported in [7], but the maximum values of $\mathrm{S}$ are around 70 $\mathrm{mW} / \mathrm{m}^{2}$, and $100 \mathrm{~mW} / \mathrm{m}^{2}$, respectively.

As far as the biological effects of electromagnetic field exposure are concerned, results presented in literature are not conclusive, some studies stating that measurable changes in brain function and behavior occur at levels associated with new technologies, including cell phone use. Cell phone radiation can change brainwave activity at levels of SAR as low as 0.1 $\mathrm{W} / \mathrm{kg}$, in comparison to the US allowable level of $1.6 \mathrm{~W} / \mathrm{Kg}$ (in $1 \mathrm{~g}$ of tissue) and ICNIRP allowable level of $2.0 \mathrm{~W} / \mathrm{Kg}$ (in $10 \mathrm{~g}$ of tissue) [9]. Cell phone radiation can affect memory and learning. Most studies only look at short-term effects, so the long-term consequences of exposures are not established, but preventative action should be taken in order to reduce exposures, particularly for vulnerable groups such as children [9]. Some other studies reviewed in [7] conclude that the balance of epidemiologic evidence indicates that mobile phone use of less than 10 years does not pose any increased risk of brain tumor or acoustic neuroma. For long-term use the conclusions are uncertain and tentative. From the available 
data it does appear that there is no increased risk for brain tumors in long-term users, with the exception of acoustic neuroma for which there is some evidence of an association.

Of particular interest is the usage of mobile phone by children. While no specific evidence exists, children or adolescents may be more sensitive to RF field exposure than adults. To date no epidemiologic studies on children are available.

Studies on neurological effects and reproductive effects have not indicated any health risks at exposure levels below the ICNIRP-limits established in 1998 [7].

Taking into account the values measured in Timisoara and presented above, values much smaller than the maximum limits given the in the ICNIRP guide and in the IEEE standard, there seems to be no threat to population health in this region.

\section{Conclusions}

Measurements of the average and maximum values of the electric field $\mathrm{E}$, magnetic field $\mathrm{H}$ and total power density $\mathrm{S}$ at $0.9,1.8$ and $2.4 \mathrm{GHz}$ were collected in the 210 nodes of a grid covering the city of Timisoara, Romania. The maps presenting the spatial distribution of the mean and maximum power density and of the mean electric and magnetic fields, were built. Results were compared to the maximum limits given the in the ICNIRP guide and in the IEEE standard, and to other results reported in literature. Possible risks on population safety were discussed. The values measured were found to pose no threat to population health in this region.

\section{Acknowledgement:}

This work was supported by a grant of the West University of Timisoara, Physics Faculty, project number UVT-FIZ-POSTDOC- 2013. The authors thank Dr. Alina Satmari and Dr. Laurentiu Artugyan from the Dept. of Geography, West University of Timisoara, for their help with the digital map of Timisoara.

\section{References}

[1] V. Khurana, L. Hardell, J. Everaert, A. Bortkiewicz, M. Carlberg, M. Ahonen, International Journal of Occupational and Environmental Health 16 (2010) 263-267

[2] M. Blettner, B. Schlehofer, J. Breckenkamp, B. Kowall, S. Schmiedel, U. Reis, P. Potthoff, J. Schüz, G. Berg-Beckhoff, Occup. Environ. Med.66 (2009) 118-123

[3] G. Berg-Beckhoff, M. Blettner, B. Kowall, J. Breckenkamp, B. Schlehofer, S. Schmiedel, C. Bornkessel, U. Reis, P. Potthoff, J. Schüz, Occup. Environ. Med.66 (2009) 124-130

[4] International Commission on Non-Ionizing Radiation Protection, ICNIRP Guidelines For 
Limiting Exposure To Time-Varying Electric, Magnetic And Electromagnetic Fields (up to $300 \mathrm{GHz}$ ), Health Physics 74 (4) (1998) 494-522

[5] CA. Gotway, RB. Ferguson, G W. Hergert and TA. Peterson, Soil Science Society of America Journal, 60, 4 (1995) 1237-1247

[6] IEEE Standard for Safety Levels with Respect to Human Exposure to RF Electromagnetic Fields, 3kHz to 300GHz, IEEE Std. C95.1-1999

[7] European commission, Scientific Committee on Emerging and Newly Identified Health Risks SCENIHR - Possible effects of Electromagnetic Fields (EMF) on Human Health (2007)

[8] S. Miclaus, P. Bechet, Rom. Journ. Phys., 52, 3- 4 (2007) 429-440

[9] L. Hardell, C. Sage, Biomedicine \& Pharmacotherapy 62 (2008) 104e109 